\title{
AVALIAÇÃO DO CONHECIMENTO DE JOGADORES DE FUTEBOL DE NOVA FRIBURGO-RJ E MOSSORÓ-RN SOBRE TRAUMATISMO DENTÁRIO.
}

\section{EVALUATION OF KNOWLEDGE OF SOCCER PLAYERS FROM NOVA FRIBURGO-RJ AND MOSSORÓ-RN ON DENTAL TRAUMA.}

\author{
Mércia Rozângela da Costa Marinho* \\ Simone Carvalho Levy** \\ Lívia Azeredo Alves Antunes"** \\ Fernanda Volpe de Abreu**** \\ Leonardo Santos Antunes*****
}

\section{RESUMO}

\begin{abstract}
A crescente prática de esportes de contato tem aumentado de forma significativa a ocorrência do traumatismo dentário. O objetivo do presente estudo é avaliar o nível de conhecimento de jogadores de futebol, no nordeste e sudeste do país, sobre o traumatismo dentário, além de sua percepção quanto às formas de prevenção. A amostra foi constituída de 53 jogadores, entre 19 e 42 anos, sendo 24 de Nova Friburgo (RJ) e 29 de Mossoró $(\mathrm{RN})$, tendo sido aplicado um questionário estruturado. Observou-se que, dos jogadores entrevistados, 91,7\% em Nova Friburgo-RJ e 48,3 \% em Mossoró-RN nunca haviam recebido qualquer tipo de informação a respeito de traumatismo dentário e que a maioria não sabia como preveni-lo (87,5\% em Nova Friburgo-RJ e 79,3 em Mossoró-RN). Quanto à conduta perante um dente avulsionado, a maioria o levaria para o dentista $(70,8 \%$ em Nova Friburgo-RJ e 79,3\% em Mossoró-RN), sendo que o meio de transporte mais citado foi a sacola ou o papel (50\% em Nova Friburgo-RJ e 37,9\% em Mossoró-RN). Quanto ao uso do protetor bucal, a quase totalidade o conhecia (91,7\% em Nova Friburgo-RJ e 79,3\% em Mossoró-RN), mas poucos o utilizavam (12,5\% em Nova Friburgo-RJ e 17,2\% em Mossoró-RN). Concluiu-se que ainda existe uma grande deficiência no conhecimento referente ao tema por parte dos jogadores de futebol, assim como de suas condutas preventivas, sendo necessário um trabalho maior dos dentistas na divulgação dos cuidados necessários para a prevenção.

DESCRITORES: Traumatismos dentários • Protetores bucais • Saúde bucal • Conhecimento • Prevenção.
\end{abstract}

\section{ABSTRACT}

The growing practice of contact sports has increased significantly the occurrence of dental injuries. The aim of this study is to evaluate the level of knowledge of football players in the northeast and southeast of the country, about dental injuries, and their perception regarding prevention methods. The sample consisted of 53 players, between 19 and 42 years, 24 of Nova Friburgo (RJ) and 29 Mossoró (RN) and applied a structured questionnaire. It was observed that among the players interviewed, (91.7\% in Nova Friburgo-RJ and $48.3 \%$ in Mossoró-RN) had never received any information about dental injuries and that most did not know how to prevent it (87 5\% in Nova Friburgo-RJ and 79.3 in Mossoró-RN). As for conduct about avulsed tooth front, most would take him to the dentist (70.8\% in Nova Friburgo-RJ and $79.3 \%$ in Mossoró-RN), and the transportation was the most cited paper or bag (50\% in New Fribourg-RJ and 37.9\% in Mossoró-RN). Regarding to the use of the mouthguard, almost all knew (91.7\% in Nova Friburgo-RJ and $79.3 \%$ in Mossoró-RN), but few of used it $(12.5 \%$ in Nova Friburgo and RJ-17 2\% in Mossoró-RN). It was concluded that there is still a great deficiency in knowledge on the topic by the football players, as well as its preventive measures requiring a larger work of dentists in the dissemination of the necessary care for their prevention.

DESCRIPTORS: Tooth injuries • Mouth protectors $\bullet$ Oral health • Knowledge $\bullet$ Prevention.

\footnotetext{
* Graduada em Odontologia pela Faculdade de Odontologia da Universidade Federal Fluminense (FOUFF/NF), Nova Friburgo, Rio de Janeiro, Brasil. mercia_cmarinho@hotmail.com

** Especialista em Odontopediatria pela Universidade Federal do Rio de Janeiro (FO/UFRJ/RJ), Rio de Janeiro, Brasil. simone-levy@ hotmail.com

*** Doutora em Odontopediatria, Professora Adjunta da Faculdade de Odontologia da Universidade Federal Fluminense (FOUFF/NF), Nova Friburgo, Rio de Janeiro, Brasil. liviaazeredo@gmail.com

**** Doutora em Odontopediatria, Professora Adjunta da Faculdade de Odontologia da Universidade Federal Fluminense (FOUFF/NF), Nova Friburgo, Rio de Janeiro, Brasil.fvolpe07@id.uff.br

***** Doutor em Ciências Médicas, Professor Adjunto da Faculdade de Odontologia da Universidade Federal Fluminense (FOUFF/NF), Nova Friburgo, Rio de Janeiro, Brasil. leonardoantunes@id.uff.br
} 


\section{N T RO DUÇÃO}

É comum vincular a cárie dentária como principal problema de saúde bucal, tanto quando se refere à prevenção quanto ao tratamento. Na verdade, essa visão é errônea, pois a Odontologia não se resume somente a esse problema. Ao longo dos anos a cárie dentária sofreu um declínio significativo em sua prevalência ${ }^{1}$ e, paralelamente a essa redução, altos índices de violência, acidentes de trânsito e uma maior participação das crianças e adolescentes em atividades esportivas contribuíram para transformar o traumatismo dentário em um problema crescente em saúde pública².

Segundo Peterson et al. ${ }^{3}$ (2005), o traumatismo dentário, juntamente com a cárie, a doença periodontal e o câncer bucal já podem ser considerados como um dos principais problemas de saúde pública. Ao mesmo tempo em que atinge o indivíduo na sua integridade física, apresenta um agravante, a partir do momento em que interfere negativamente a nível psicológico, podendo ser decisivo no comprometimento do convívio social ${ }^{4}$, gerando impacto na qualidade de vida ${ }^{5}$.

No caso de um traumatismo dentário ou de um impacto mais grave, é de extrema importância um atendimento imediato, para que seja possível obter um melhor prognóstico. No momento do acidente, o envolvido deve demonstrar total segurança quanto à conduta correta; é importante tentar preservar ao máximo o elemento ou fragmento dentário, no menor tempo possível, e imediatamente procurar um cirurgião-dentista. Vale lembrar que o acompanhamento do paciente, mesmo com o tratamento finalizado, é indispensável ${ }^{6}$.

O uso de protetores bucais foi a solução encontrada pelos cirurgiões-dentistas para minimizar o número de lesões orofaciais decorrentes do trauma durante práticas esportivas de contato $^{7}$. No entanto, apesar de serem considerados tão vantajosos, ainda são dispositivos desconhecidos por grande parte da população, inclusive por profissionais da Odontologia e ligados ao esporte ${ }^{8}$.

Uma questão preocupante são as atitudes dos atletas diante das situações de traumatismo. O desconhecimento e o despreparo para realizar os procedimentos de urgência adequados podem acarretar uma conduta inadequada. Dessa forma, o presente estudo teve como objetivo, em um primeiro momento, realizar uma investigação para traçar a atitude de jogadores de futebol a respeito da conduta imediata perante um traumatismo dentário, assim como sua percepção sobre as formas de prevenção.

\section{MATERIAIS E MÉTODOS}

Este estudo foi submetido ao Comitê de Ética em Pesquisa do Hospital Universitário Antônio Pedro da Universidade Federal Fluminense, RJ, e aprovado sob número CEP- 247/11, obedecendo aos aspectos éticos concernentes à pesquisa com seres humanos.

Trata-se de uma pesquisa transversal de caráter investigativo, realizado com uma amostra de conveniência formada por 53 jogadores de futebol profissional, selecionados no município de Mossoró, Rio Grande do Norte $(n=29)$ e no município de Nova Friburgo, Rio de Janeiro $(n=24)$.

Este estudo consistiu de uma amostragem de conveniência, uma vez que se caracterizou por não existir a preocupação com o desenho de um plano particular de amostragem. O objetivo não foi generalizar conclusões, pelo potencial viés de seleção, senão descrever as características principais do grupo de estudo ${ }^{9}$.

Como critério de inclusão, foram selecionados jogadores profissionais de futebol com vínculo em clubes.

Primeiramente, realizou-se o contato com os diretores dos clubes de futebol a respeito da pesquisa. Após o consentimento destes, foram dadas instruções aos esportistas participantes sobre a melhor forma de preenchimento dos questionários. Estes foram respondidos pelos atletas durante dias de treinamento.

$\mathrm{O}$ instrumento utilizado nesta pesquisa foi um questionário autoaplicável estruturado, contendo informações a respeito das características demográficas do atleta (idade, cidade e escolaridade), da sua atitude diante do traumatismo dentário, bem como do conhecimento a respeito de protetores bucais.
MARINHO MRC LEVY SC

ANTUNES LAA

ABREU FV,

ANTUNES LS

AVALIAÇÃO DO

CONHECIMENTO

DE JOGADORES

DE FUTEBOL DE

Nova FRIBUR-

GO-RJ E MOS-

SORÓ-RN SOBRE

TRAUMATISMO

DENTÁRIO.

189
ReV. OdOnTOL.

Univ. CID. São

PAulo

2013; 25(3): 188 -

95, SET-DEZ 
MARINHO MRC LEVY SC ANTUNES LAA ABREU FV, ANTUNES LS

AVALIAÇÃO DO CONHECIMENTO DE JOGADORES DE FUTEBOL DE NOVA FRIBURGO-RJ E MOSSORÓ-RN SOBRE

TRAUMATISMO DENTÁRIO.
ISSN 1983-5183

Para a análise quantitativa dos resultados, os dados foram tabulados em um programa estatístico SPSS (Statistical Package for Social Science) versão 17.0, com digitação única, propiciando à pesquisa uma maior fidedignidade e confiabilidade. Utilizou-se a frequência relativa e absoluta como metodologia estatística para posterior discussão dos resultados. Foi aplicado, ainda, o teste qui-quadrado (x2) em nível de $5 \%$ de significância $(p<0,05)$, para se estabelecer relação entre variáveis de interesse.

\section{RESULTADOS}

Foram entrevistados 53 jogadores de futebol, entre 19 e 42 anos, sendo 24 de Nova Friburgo-RJ e 29 de Mossoró-RN. Os dados relativos à média de idade e es- colaridade de cada grupo encontram-se expressos na Tabela 1.

Na Tabela 2, pode-se observar que, dos jogadores de futebol entrevistados, $91,7 \%$ em Nova Friburgo-RJ e 48,3 \% em Mossoró-RN nunca haviam recebido algum tipo de informação a respeito de traumatismo dentário e que a maioria não sabia como preveni-lo (87,5\% em Nova Friburgo-RJ e 79,3 em Mossoró-RN). Quanto à conduta perante um dente avulsionado, a maioria levaria o dente para o dentista (70,8\% em Nova Friburgo-RJ e 79,3\% em Mossoró-RN), sendo que o meio de transporte mais citado foi a sacola ou papel $(50 \%$ em Nova Friburgo-RJ e 37,9\% em Mossoró-RN). Quase todos os entrevistados consideravam que o atendimento deveria ser realizado imediatamente após o trauma-

\begin{tabular}{llll}
\hline \hline Média de idade (DP) & FRIBURGO, RJ & MOSSORÓ, RN & TOTAL \\
Escolaridade $(\%)$ & $24,8(5,2)$ & $25,79(6,8)$ & - \\
Fundamental incompleto & - & $3(10,3)$ & $3(5,7)$ \\
Fundamental completo & $2(8,3)$ & $4(13,8)$ & $6(11,3)$ \\
Médio incompleto & $3(12,5)$ & $2(6,9)$ & $5(9,4)$ \\
Médio completo & $14(58,3)$ & $16(55,2)$ & $30(56,6)$ \\
Superior incompleto & $5(20,8)$ & $1(3,4)$ & $6(11,3)$ \\
Superior completo & - & $3(10,3)$ & $3(5,7)$ \\
Total & $24(100)$ & $29(100)$ & $53(100)$ \\
\hline \hline
\end{tabular}

Tabela 1 - Caracterização da amostra.

\begin{tabular}{|c|c|c|c|c|}
\hline & FRIBURGO, RJ & MOSSORÓ, RN & TOTAL & P-VALOR \\
\hline \multicolumn{5}{|c|}{ Recebimento de informação a respeito de traumatismo dentário n(\%) } \\
\hline Sim & $2(8,3)$ & $15(51,7)$ & $17(32,1)$ & \multirow{2}{*}{$<0,01$} \\
\hline Não & $22(91,7)$ & $14(48,3)$ & $36(67,9)$ & \\
\hline \multicolumn{5}{|c|}{ Possui conhecimento sobre como se previne o traumatismo dentário } \\
\hline Sim & $3(12,5)$ & $6(20,7)$ & $9(17)$ & \multirow{2}{*}{0,34} \\
\hline Não & $21(87,5)$ & $23(79,3)$ & $44(83)$ & \\
\hline \multicolumn{5}{|c|}{ Conduta diante de um dente avulsionado } \\
\hline Levaria para o dentista & $17(70,8)$ & $23(79,3)$ & $40(75,5)$ & \multirow{4}{*}{-} \\
\hline Recolocaria o dente no local & $4(16,7)$ & - & $4(7,5)$ & \\
\hline Jogaria o dente fora & - & $2(6,9)$ & $2(3,8)$ & \\
\hline Não sabe o que fazer & $3(12,5)$ & $4(13,8)$ & $7(13,2)$ & \\
\hline \multicolumn{5}{|c|}{ Meio de transporte ideal para o dente avulsionado } \\
\hline Boca & $3(12,5)$ & $2(6,9)$ & $5(9,4)$ & \multirow{5}{*}{0,18} \\
\hline Líquido & $4(16,7)$ & $5(17,2)$ & $9(17)$ & \\
\hline Papel/sacola & $12(50)$ & $11(37,9)$ & $23(43,4)$ & \\
\hline Outros & $3(12,5)$ & $1(3,4)$ & $4(7,5)$ & \\
\hline Não sabe & $2(8,3)$ & $10(34,5)$ & $12(22,6)$ & \\
\hline \multicolumn{5}{|c|}{ Tempo ideal para atendimento pós traumatismo dentário } \\
\hline Imediatamente & $23(95,8)$ & $24(82,8)$ & $47(88,7)$ & \multirow{2}{*}{0,14} \\
\hline Não soube informar & $1(4,2)$ & $5(17,2)$ & $6(11,3)$ & \\
\hline
\end{tabular}

REV. ODONTOL. UNIV, CID, SÃO PAULO 2013; 25(3): 188 95, SET-DEZ

Tabela 2 - Conhecimentos e atitudes dos jogadores de futebol perante o traumatismo. 
tismo dentário (95,8 em Nova Friburgo-RJ e 82,8 em Mossoró-RN). Ao se relacionar as respostas de acordo com o município dos entrevistados, observou-se diferença significativa apenas na pergunta referente ao conhecimento dos entrevistados com relação ao traumatismo dentário $(p<0,01)$.

O Gráfico 1 demonstra a percepção dos jogadores de futebol quanto ao uso do protetor bucal. Destaca-se que quase a totalidade dos entrevistados o conhecia $(91,7 \%$ em Nova Friburgo-RJ e $79,3 \%$ em Mossoró-RN), poucos o utilizavam $(12,5 \%$ em Nova Friburgo-RJ e $17,2 \%$ em Mossoró-RN) e a maioria considerava seu uso importante na prática de esportes (83,3\% em Nova Friburgo-RJ e 89,7\% em Mossoró-RN).

\section{I SCUSSÃO}

A prática de esportes é um dos principais fatores responsáveis pelos altos índices de lesões orofaciais, por ser uma atividade na qual os atletas estão mais sujeitos a sofrer um possível trauma, que pode influenciar no seu desempenho e na sua qualidade de vida.

Com o intuito de minimizar as sequelas produzidas por esses acidentes, observa-se a necessidade, por parte dos cirurgiões-dentistas, de lançar mão de medidas de prevenção, entre elas o protetor bu$\mathrm{cal}^{10}$. Contudo, são raros os levantamentos epidemiológicos acerca do conhecimento relacionado ao traumatismo dentário e à utilização do protetor bucal como método de proteção entre esportistas.

A preocupação com a saúde bucal dos atletas tem sido manifestada nos últimos anos pela tentativa de alguns cirurgiões-dentistas com visão esportista, em tornar a Odontologia Desportiva reconhecida ${ }^{11}$. Essa é uma área da Odontologia recente e pouco divulgada, na qual atuam cirurgiões-dentistas dispostos a melhorar o rendimento dos atletas através da manutenção da saúde oral, tratando e prevenindo lesões originadas de práticas esportivas. Nesse novo contexto, o dentista atua em uma equipe multidisciplinar, visando detectar fatores prejudiciais, como: respiração bucal e posicionamento de dentes de forma inadequada ${ }^{12,13}$.

Diversos trabalhos indicam que os altos índices de traumatismos em esportes o variam com o esporte praticado, sendo esta uma das razões para ter sido desenvolvido o presente estudo ${ }^{14,15}$. No entanto, a frequência dos acidentes provocados na face, principalmente os relacionados aos dentes durante o ato esportivo, é muito maior do que o número divulgado ${ }^{16}$. Esse tipo de trauma representa o terceiro atendimento mais procurado relacionado a traumas de face ${ }^{17}$.

O futebol é um esporte predominan-
MARINHO MRC LEVY SC ANTUNES LAA ABREU FV, ANTUNES LS

AVALIAÇÃO DO CONHECIMENTO DE JOGADORES DE FUTEBOL DE NOVA FRIBURGO-RJ E MOSSORÓ-RN SOBRE TRAUMATISMO DENTÁRIO.
Gráfico 1 - Percepção dos jogadores de futebol quanto ao uso do protetor bucal
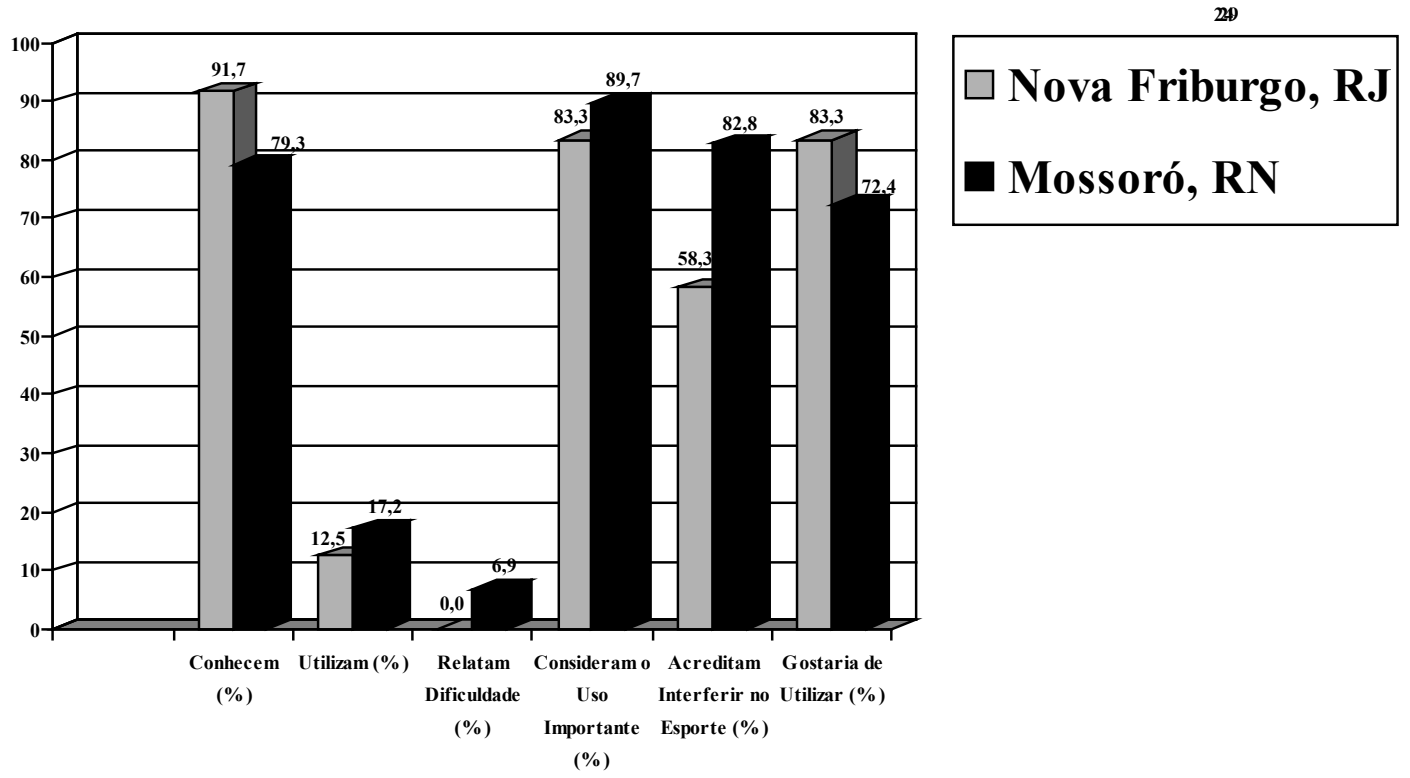

Nota: *Teste do Qui-quadrado ou Exato de Fisher; valor em negrito indica significância estatística ( $\mathrm{p} \leq 0.05)$.
REV, ODONTOL. UNIV, CID, SÃO PAULO

$2013 ; 25(3): 188-$ 95, SET-DEZ 
MARINHO MRC LEVY SC

ANTUNES LAA

ABREU FV,

ANTUNES LS

AVALIAÇÃO DO

CONHECIMENTO

DE JOGADORES

DE FUTEBOL DE

NOVA FRIBUR-

GO-RJ E MOS-

SORÓ-RN SOBRE

TRAUMATISMO

DENTÁRIO.

$192 \ldots$

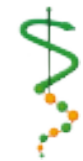

REV. ODONTOL. UNIV. CID, SÃO

PAULO

2013; 25(3): 188-

95, SET-DEZ

I SSN 1983-5183

temente masculino, por isso o acesso a clubes femininos torna-se mais difícil. O grupo estudado foi caracterizado por ser constituído por integrantes exclusivamente do sexo masculino, contexto semelhante ao estudo de Santana ${ }^{18}$ (2012), no qual a grande maioria da população (98\%) participante foi de homens.

Diferentemente de outras situações que podem levar a acidentes orofaciais, os traumatismos dentários ocasionados no esporte possuem uma particularidade, pois podem ser prevenidos a partir da instalação de dispositivos bucais individuais, responsáveis por uma redução considerável na incidência de sua ocorrência, além de diminuírem a extensão e a gravidade das lesões ${ }^{19}, 20$. Embora exista a possibilidade de prevenção, os resultados da pesquisa comprovaram que esta ainda é uma realidade distante no esporte.

Nos Estados Unidos, a obrigatoriedade quanto ao uso do protetor bucal em diversas modalidades esportivas já é uma realidade desde a década de 50, resultado de campanhas de conscientização de profissionais do esporte, da saúde e da educação ${ }^{13}$. Em contrapartida, no Brasil, ainda são poucos os esportes que regulamentam como obrigatório o uso de protetores durante as competições, já que a Odontologia direcionada ao esporte ainda é bastante ignorada ${ }^{11}$.

No estudo desenvolvido por Ferrari et al. ${ }^{21}(2000)$, os atletas citaram o futebol como um dos esportes que mais ocasionaria traumas dentários $(23,1 \%)$, perdendo somente para o basquete $(37,7 \%)$; handebol $(37,8 \%)$ e jiu-jitsu (41,1\%). Entretanto, de acordo com os resultados do mesmo autor, a quantidade de esportistas que utilizam protetor bucal em cada um desses esportes ainda é preocupante: no futebol $(1,4 \%)$; no basquete $(2,2 \%)$; no handebol $(4,1 \%)$; no judô $(7,4 \%)$; no jiu-jitsu $(19,6 \%)$ e no hóquei $(92,3 \%)$. Isso pode ser justificado pela falta de obrigatoriedade do uso do dispositivo de proteção principalmente nos esportes de contato, sendo o boxe o único em que é obrigatório.

Neste estudo, 20,8\% dos jogadores de futebol de Nova Friburgo-RJ relataram já ter sofrido traumatismos orofaciais e tais dados vão ao encontro de Yamada et al. ${ }^{22}$
(1998) que apontam 32,3\% de ocorrência de traumatismos. Quanto aos jogadores de Mossoró-RN, 62,1\% afirmaram ter sofrido trauma. Embora seja evidente a importância do protetor bucal no papel de prevenção, não pôde ser observada correlação direta, pelo menos nesta pesquisa, do uso de tal dispositivo com a diminuição da ocorrência de acidentes, visto que os baixos índices relacionados ao seu uso pelos entrevistados tanto do Rio de Janeiro $(12,5 \%)$ como no Rio Grande do Norte $(17,2 \%)$ não justificam os resultados obtidos quanto à ocorrência de trauma em ambos os estados. Além disso, apenas 8,3\% dos entrevistados de Nova Friburgo-RJ citaram o protetor bucal como forma de prevenção de possíveis complicações na região bucal. Enquanto no estado potiguar, o mesmo não foi citado por nenhum membro dos times, apesar de alguns jogadores afirmarem utilizar o protetor durante a prática esportiva, o protetor durante a prática esportiva. Esse resultado contraditório demonstra que os atletas usam o protetor sem mesmo saberem a sua real função e importância, o que comprova uma falta de informação.

O reimplante dentário imediato tem sido amplamente aceito como um meio efetivo de tratamento de dentes avulsionados, sendo o meio de conservação e o tempo de permanência fora do alvéolo fatores determinantes para o sucesso terapêutico ${ }^{14,23}$. Embora não surpreenda, no estudo realizado por Santana ${ }^{18}$ (2012), quando questionados sobre o que fariam com o dente avulsionado, observou-se o baixo índice de respostas positivas em relação ao reimplante do dente no alvéolo logo após o trauma $(82,8 \%$ esperariam mais de trinta minutos para reimplantar o dente ou não o fariam). Essa situação pode ser entendida como insegurança por parte dos leigos em manusear o dente avulsionado em direção ao alvéolo no momento do acidente ou por muitas vezes já o considerarem condenado, atitudes decorrentes da falta de conhecimento, além de ser vista como uma forma incomum de tratamento. Um exemplo disso foi a resposta dada por alguns dos participantes da pesquisa, ao afirmarem que jogariam fora o elemento dentário nesses casos, demonstrando, as- 
sim, o quanto desconhecem a dimensão da importância do dente, que ainda pode ser recuperado.

Na literatura existe unanimidade quando o assunto são as vantagens do reimplante imediato; no entanto, o que se observa é que geralmente os dentes avulsionados não são encontrados ou são mantidos inadequadamente. Essa questão também foi apresentada por Guedes et al. ${ }^{24}$ (2012), segundo os quais $89,08 \%$ dos dentes avulsionados receberam implante tardio.

De acordo com o presente estudo, pôde ser observado que os resultados são compatíveis com a literatura, pois a grande maioria respondeu positivamente ao atendimento imediato, semelhante, por exemplo, ao encontrado por Granville-Garcia et al. ${ }^{25}$ (2007) onde $86,1 \%$ consideraram que o atendimento poderia ser imediato e 13,9\% responderam que apenas após a hemostasia. Os resultados obtidos nesses estudos demonstraram que, apesar da maioria dos entrevistados considerarem o atendimento imediato de extrema impor- tância, não é o que ocorre na prática. $\mathrm{E}$ independente da região do país (nordeste ou sudeste), não foi isso que determinou diferenças no nível de conhecimento a respeito dos protetores bucais e na conduta diante de um traumatismo dentário.

Tendo-se em vista que a atividade esportiva é um fator predisponente ao traumatismo, e estando os atletas inevitavelmente expostos aos riscos de acidentes rotineiramente, a inclusão de programas educativo-preventivos junto à população torna-se necessária em qualquer região do país. Cabe ao cirurgião-dentista assumir a responsabilidade de orientar e incentivar os esportistas e, de certa forma, a população em geral, acerca da importância de medidas preventivas relacionadas ao traumatismo dentário.

Com base nos resultados encontrados, este estudo servirá de subsídio para elaboração de prioridades de ação preventiva e curativa, a fim de se buscar uma melhora na qualidade de vida da população envolvida.
MARINHO MRC LEVY SC

ANTUNES LAA

ABREU FV,

ANTUNES LS

AVALIAÇÃO DO CONHECIMENTO DE JOGADORES

DE FUTEBOL DE

NOVA FRIBUR-

GO-RJ E MOS-

SORÓ-RN SOBRE

tRAUMATISMO

DENTÁRIO.








REV. ODONTOL. UNIV, CID, SÃO PAULO 2013; 25(3): 188 95, SET-DEZ
1. Bonecker M, Cleaton-Jones P. Trends in dental caries in Latin American and Caribbean 5-6- and 11-13-year-old children: a systematic review. Community Dent Oral Epidemiol 2003 Apr;31(2):152-7.

2. Marcenes W, al Beiruti N, Tayfour D, Issa S. Epidemiology of traumatic injuries to the permanent incisors of 9-12-year-old schoolchildren in Damascus, Syria. Endod Dent Traumatol 1999 Jun; 15(3):117-23.

3. Petersen PE, Bourgeois D, Ogawa $H$, Estupinan-Day S, Ndiaye C. The global burden of oral diseases and risks to oral health. Bull World Health Organ 2005 Sep;83(9):661-9.

4. Silveira EG, Araújo SM, Schmitt BHE, Farias MMAG, Campos L, Caregnato M. Conhecimento e atitudes dos odontopediatras do Estado de Santa Catarina acerca de mecanismos de prevenção de traumatismos bucais relacionados a esportes. Rev odontol UNESP (Online) 2009 nov-dez ;38(6):341-6.

5. Antunes LAA, Leão AT, Maia LC. Impacto do traumatismo dentário na qualidade de vida de crianças e adolescentes: revisão crítica e instrumentos de medida. Ciênc saúde coletiva 2012 dez; 17(12):3417-24.

6. Kramer P, Feldens $\mathrm{C}$. Traumatismo na dentição decídua: prevenção, diagnóstico e tratamento. São Paulo: Santos; 2005.

7. Westerman B, Stringfellow PM, Eccleston JA. EVA mouthguards: how thick should they be? Dent Traumatol 2002 Feb;18(1):24-7.

8. Sizo SR, Silva ES, Rocha MPdC, Klautau EB. Avaliação do conhecimento em odontologia e educação física acerca dos protetores bucais. Rev Bras Med Esporte 2009 ago; 15(4):282-6.

9. Luiz R, Tânia G, Magnanini M. Planejamento amostral. In: Luiz R, Costa A, Nadanovsky P. Epidemiologia e bioestatística na pesquisa odontológica. São Paulo: Atheneu; 2005. p. 245-72.
10. Knapik JJ, Marshall SW, Lee RB, Darakjy SS, Jones SB, Mitchener TA, et al. Mouthguards in sport activities : history, physical properties and injury prevention effectiveness. Sports Med 2007 37(2):117-44.

11. Costa SS. Odontologia desportiva na luta pelo reconhecimento. Rev odontol Univ Cid São Paulo 2009 dez;21(2):162-8.

12. Forsberg CM, Tedestam G. Etiological and predisposing factors related to traumatic injuries to permanent teeth. Swed Dent J 1993 17(5):183-90.

13. Santiago E, Simões R, Soares D, Pereira JA, Caldas T. Protector bucal "Custom-Made" : indicações, confecção e características essenciais. Arq Med 2008 22(1):25-33.

14. Andreasen JO, Andreasen FM. Textbook and color atlas of traumatic injuries to the teeth. 3. ed. Copenhagen: Munksgaard; 1994.

15. Sane J, Ylipaavalniemi P. Dental trauma in contact team sports. Endod Dent Traumatol 1988 Aug;4(4):164-9.

16. Padilla R, Balikov S. Sports dentistry: coming of age in the '90s. J Calif Dent Assoc 1993 Apr;21(4):27-34, 6-7.

17. Andreasen FM, Andreasen JO. Diagnosis of luxation injuries: the importance of standardized clinical, radiographic and photographic techniques in clinical investigations. Endod Dent Traumatol 1985 Oct; 1(5):160-9.

18. Santana NM. Avaliação do conhecimento sobre a conduta emergencial do trauma dental de profissionais do futebol em Curitiba, Brasil [Dissertação]. Curitiba: Universidade Federal de Curitiba; 2012.

19. Ferreira R. Impacto radical. Rev Assoc Paul Cir Dent 1998 52(4):265-71.

20. Futaki J, Motta LFG. Protetores bucais: promoção da saúde na odontologia. Rev Odontol Univ St Amaro 2000 jul-dez;5(2):98-105. 
21. Ferrari CH, Simi JRJ, Medeiros JMF. Ocorrência de traumatismo dental $e$ nível de esclarecimento e uso de protetor bucal em diferentes grupos de esportistas2000. Disponível m: http:// www.odontologia.com.br/artigos. asp? $i d=88 \& i \% 20 d e s p=2 \& l e r=s$.

22. Yamada T, Sawaki Y, Tomida S, Tohnai I, Ueda M. Oral injury and mouthguard usage by athletes in Japan. Endod Dent Traumatol 1998 Apr;14(2):84-7.

23. Stokes AN, Anderson HK, Cowan TM. Lay and professional knowledge of methods for emergency management of avulsed teeth. Endod Dent Traumatol 1992 Aug;8(4):160-2.
24. Guedes AO, Estrela C, Alencar GAH. Estudo epidemiológico de 261 dentes permanentes avulsionados de pacientes tratados em um serviço de urgência odontológica [Tese]. Goiás Universidade Federal de Goiás 2012.

25. Granville-Garcia AF, Lima EM, Santos PG, Menezes VA. Avaliação do conhecimento de professores de educação física de Caruaru-PE sobre avulsão-reimplante. Pesq Bras Odontoped Clin Integr 2007 jan-abr ;7(1):15-20.

Recebido em 15-04-2013

Aceito em 19-08-2013
MARINHO MRC

LEVY SC

ANTUNES LAA

ABREU FV,

ANTUNES LS

AVALIAÇÃO DO

CONHECIMENTO

DE JOGADORES

DE FUTEBOL DE

NOVA FRIBUR-

GO-RJ E MOS-

SORÓ-RN SOBRE

TRAUMATISMO

DENTÁRIO.

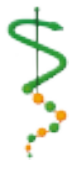

REV, ODONTOL,

UNIV, CID, SÃO

PAULO

$2013 ; 25(3): 188-$

95, SET-DEZ 